\title{
Myelin Detection Using Rapid Quantitative MR Imaging Correlated to Macroscopically Registered Luxol Fast Blue-Stained Brain Specimens
}

\author{
(D).B.M. Warntjes, (D)A. Persson, (D). Berge, and (DW. Zech
}

\begin{abstract}
BACKGROUND AND PURPOSE: Myelin detection is of great value in monitoring diseases such as multiple sclerosis and dementia. However, most MR imaging methods to measure myelin are challenging for routine clinical use. Recently, a novel method was published, in which the presence of myelin is inferred by using its effect on the intra- and extracellular water relaxation rates and proton density, observable by rapid quantitative MR imaging. The purpose of this work was to validate this method further on the brains of 12 fresh, intact cadavers.
\end{abstract}

MATERIALS AND METHODS: The 12 brains were scanned with a quantification sequence to determine the longitudinal and transverse relaxation rates and proton density as input for the myelin estimations. Subsequently, the brains were excised at postmortem examination, and brain slices were stained with Luxol fast blue to verify the presence of myelin. The optical density values of photographs of the stained brain slices were registered with the MR images and correlated with the myelin estimation performed by quantitative MR imaging.

RESULTS: A correlation was found between the 2 methods with a mean Spearman $\rho$ for all subjects of $0.74 \pm 0.11$. Linear regression showed a mean intercept of $1.50 \% \pm 2.84 \%$ and a mean slope of $4.37 \% \pm 1.73 \% / \%$. A lower correlation was found for the separate longitudinal relaxation rates and proton density $(\rho=0.63 \pm 0.12$ and $-0.73 \pm 0.09$, respectively). For transverse relaxation rates, the $\rho$ was very low $(0.11 \pm 0.28)$.

CONCLUSIONS: The observed correlation supports the validity of myelin measurement by using the MR imaging quantification method.

ABBREVIATIONS: $L F B=$ Luxol fast blue; $O D=$ optical density; $P D=$ proton density; qMRI = quantitative MRI; $R_{1}=$ longitudinal relaxation rate; $R_{2}=$ transverse relaxation rate

$\mathbf{T}$ he measurement of myelin content in the brain is important for neurodegenerative diseases such as multiple sclerosis or dementia, in which increasing cerebral demyelination can be observed in the course of the disease. ${ }^{1-3}$ Also, for brain development, monitoring increasing myelination has clinical value. ${ }^{4,5}$ With MR imaging, there are a number of indirect measures for the detection of myelin, most of them based on multicomponent analysis of the $\mathrm{T} 2$ relaxation. In this approach, the observation of a short $\mathrm{T} 2$ relaxation component is attributed to the presence of thin layers of water trapped inside the myelin sheaths. ${ }^{6-10}$

Recently, a model has been published that infers the presence

Received October 27, 2016; accepted after revision February 3, 2017.

From the Center for Medical Image Science and Visualization (J.B.M.W., A.P., W.Z.) and Division of Cardiovascular Medicine, Department of Medical and Health Sciences (J.B.M.W.), Linköping University, Linköping, Sweden; SyntheticMR AB (J.B.M.W.), Linköping, Sweden; Institute of Forensic Medicine (J.B., W.Z.), Linköping, Sweden; and Institute of Forensic Medicine (W.Z.), University of Bern, Bern, Switzerland.

Please address correspondence to J.B.M. Warntjes, MD, Center for Medical Image Science and Visualization, Linköping University, 58185 Sweden; e-mail: marcel.warntjes@cmiv.liu.se

http://dx.doi.org/10.3174/ajnr.A5168 of myelin partial volume based on its effect on intra- and extracellular water due to magnetization exchange. ${ }^{11}$ The vicinity of myelin reduces the local longitudinal relaxation rate $\left(R_{1}\right)$, the transverse relaxation rate $\left(\mathrm{R}_{2}\right)$, and proton density $(\mathrm{PD})$. By rapid quantitative MR imaging ( $\mathrm{qMRI}$ ) of the relaxation rates and proton density, a multiparametric space is measured, forming the basis for an estimation of myelin partial volume in each acquisition voxel. Because the quantification sequence requires only $5-7$ minutes for full coverage of the brain, it may be suitable for routine clinical use.

The aim of this study was to validate the model further by correlating the estimated myelin content by qMRI with myelin estimations from photographs of brain slices that were stained with myelin-sensitive Luxol fast blue (LFB) in a postmortem application.

\section{MATERIALS AND METHODS \\ Study Group}

Twelve fresh, intact cadavers were prospectively investigated in the study, 9 men and 3 women, with a mean age $62 \pm 10$ years (range, 46-74 years). The study cases were routine forensic cases 


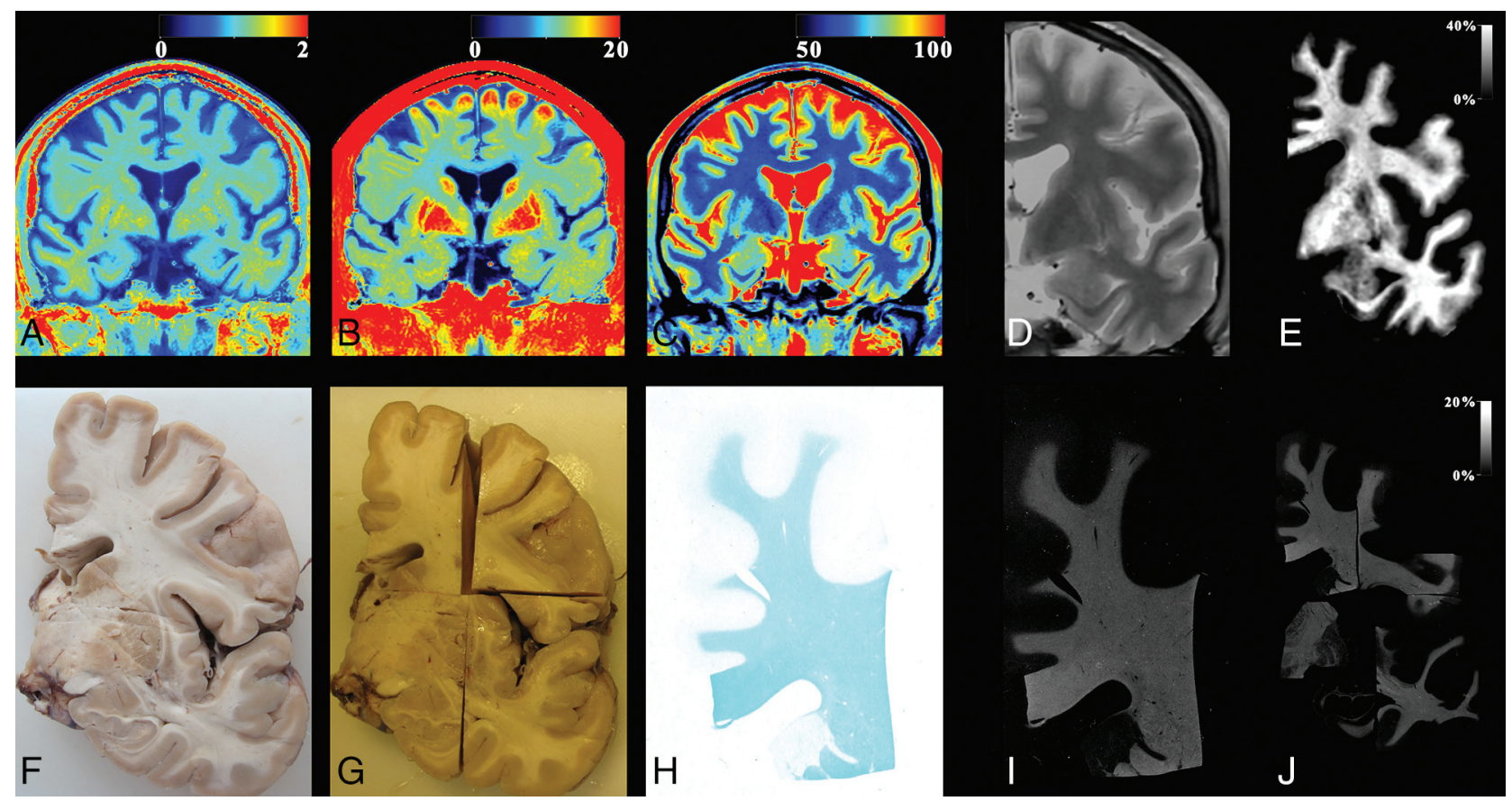

FIG 1. The process used for myelin evaluation on a male subject, 69 years of age, acquired at a temperature of $10^{\circ} \mathrm{C}$. The $\mathrm{MR}$ imaging quantification sequence provided the $R_{1}, R_{2}$, and PD maps of coronal slices of the cadaver $(A-C)$. A synthetic proton density-weighted image was created for registration purposes using the $R_{1}, R_{2}$, and PD maps as input, resampled to $0.1 \mathrm{~mm} /$ pixel ( $D$, zoomed in). The $R_{1}$ map was corrected for temperature and then used, with the original $R_{2}$ and PD maps, to generate the myelin partial volume map with the same algorithm as used for living subjects $(E)$. For the histologic images, the brain was extracted and cut into coronal slices $(F)$. Slices were fixated by using formaldehyde and cut into smaller pieces after fixation $(G)$. The separate pieces of brain slices were stained with Luxol fast blue and photographed $(H)$. The optical density of the photographs was converted to an intensity scale. These images were also resampled to $0.1 \mathrm{~mm} / \mathrm{pixel}(I)$. All pieces were registered to the synthetic proton density-weighted image ( ), so that each pixel from the slice photographs was at the same place as the corresponding MR imaging pixel. Finally, the resolution of both MR images and photographs was down-sampled to the original MR imaging resolution of $0.7 \mathrm{~mm} /$ pixel.

from the local forensic institution, in which forensic postmortem examinations were ordered by the local authorities. Cadavers were refrigerated in cooling chambers before imaging to avoid progressing putrefaction. Trauma cases and cases with known brain diseases in their medical history were excluded. Pre-MR imaging and prepostmortem information about cases was received from paramedic reports, police, relatives of the deceased, or the bureau of the district attorney. Due to the forensic nature of the cases, no further clinical data were available. MR imaging examinations and the use of the imaging data were approved by the local ethics committee. Postmortem interval (the time between death and the postmortem MRI examination) ranged from 20 hours to 3 days. During MR imaging, the cadaver core body temperatures were assessed in real-time with MR imaging-compatible temperature probes that were placed in the esophagus before the MR imaging examination. The mean core body temperature of the cadavers during acquisition was $7.8^{\circ} \pm 3.1^{\circ} \mathrm{C}$. Board-certified forensic pathologists performed postmortem examinations immediately after the MR imaging examinations. The causes of death were myocardial infarction $(n=5)$, acute cardiac arrest $(n=3)$, pulmonary embolism $(n=2)$, and internal exsanguination $(n=2)$.

\section{MR Imaging Acquisition Method}

An overview of the entire process is provided in Fig 1. The study subjects underwent a qMRI acquisition ${ }^{12}$ to measure T1 and T2 relaxation times and proton-density values. Relaxation rate $R_{1}$ corresponds to $1 / \mathrm{T} 1$; and $\mathrm{R}_{2}$, to $1 / \mathrm{T} 2$. The sequence was a saturation recovery turbo-spin-echo with a multiecho readout. Four different saturation delay times (at 170, 660, 2290, and $4740 \mathrm{~ms}$ ) and 2 different TEs (at 23 and $105 \mathrm{~ms}$ ) were acquired, resulting in a matrix of $4 \times 2=8$ images per section with different signal intensities depending on the delay time and TE. The TR was 4.9 seconds. In total, 30 sections of 4-mm thickness were acquired in the coronal plane, with an FOV of $230 \times 190 \mathrm{~mm}$, with an in-plane acquisition resolution of $0.7 \mathrm{~mm} / \mathrm{pixel}$, reconstructed at $0.45 \mathrm{~mm} /$ pixel. The scan time was 7 minutes. The MR imaging scanner was a $3 \mathrm{~T}$ Ingenia (Philips Healthcare, Best, the Netherlands) using 16-channel head/base combination. Postprocessing was performed with SyMRI 8.0 (SyntheticMR, Linköping Sweden).

\section{Temperature Correction}

Four ROIs were placed in the frontal white matter; 4 , in the anterior cingulate cortex; and 4, in the lateral ventricles of all subjects to obtain an estimate of the mean $\mathrm{T} 1$ and $\mathrm{T} 2$ relaxation times for white matter, gray matter, and CSF as a function of temperature. These data were used to derive a correction function to compensate for relaxation time differences due to the lower temperature of the subjects than $37^{\circ} \mathrm{C}$. A linear change per degree Celsius was assumed. ${ }^{13,14}$ Differences due to age, illness, or any other subjectspecific changes were ignored.

AJNR Am J Neuroradiol 38:1096-102 Jun 2017 www.ajnr.org 


\section{MR Imaging Myelin Estimation}

The temperature-corrected $\mathrm{R}_{1}, \mathrm{R}_{2}$, and $\mathrm{PD}$ maps were used as input into the myelin model as previously described. ${ }^{11}$ In summary, this model consists of 4 partial volumes per acquisition voxel: myelin partial volume, cellular partial volume, free water partial volume, and excess parenchymal partial volume, in which each partial volume has its own $\mathrm{R}_{1}, \mathrm{R}_{2}$, and PD properties. The properties of free water partial volume and excess parenchymal partial volume were fixed to the properties of CSF $\left(R_{1}=0.24\right.$ seconds $^{-1}, \mathrm{R}_{2}=0.87$ seconds $^{-1}$, and $\left.\mathrm{PD}=100 \%\right)$. The myelin partial volume $R_{2}$ was fixed to a literature value of 77 seconds ${ }^{-1}$. The remaining parameters of the model were then set by the observable $R_{1}, R_{2}$, and PD properties for each voxel in the brains of a group of healthy subjects. Subsequently, potential pathologic changes were modeled as 2 factors: a decrease of myelin partial volume and an increase of excess parenchymal partial volume, in comparison with the healthy brain. All distributions of the 4 partial volumes, ranging from $0 \%$ to $100 \%$ of the acquisition volume, generated a grid in $R_{1}, R_{2}$, and $P D$ space. The observed $R_{1}, R_{2}$, and PD combinations from the qMRI acquisition were projected onto this grid, to estimate the myelin partial volume for each voxel.

\section{Photographs of LFB-Stained Brain Slices and Histologic Examinations}

The subjects' brains were extracted at forensic postmortem and cut into 2-cm-thick coronal slices. In each case, 1 anterior slice through the head of the caudate nucleus and 1 posterior slice through the thalamus were taken for further examinations. Only the left hemisphere was included. The brain specimens were soaked in formalin for 5 days. After formalin fixation, the brain slices were further cut into 6 smaller pieces to fit the $4 \times 6 \mathrm{~cm}$ glass plates and sliced to $4-\mu \mathrm{m}$ thickness. A Luxol fast blue staining was applied on brain slices. Photographs of LFB-stained brain slices were taken in a fixed setup with a light bench for uniform backlighting of the specimens and a stative. The glass plates covered about half of the final image so that most of the photograph was exposed to the background light, keeping the white filter of the camera similar for all photographs. Zoom and focus were set to manual and kept constant. The acquired JPG image resolution was $0.02 \mathrm{~mm} /$ pixel. The photographs were decomposed into red $(\mathrm{R})$, green $(\mathrm{G})$ and blue $(\mathrm{B})$ channels with values in the range of 0-255 with a custom-built IDL program (ITT Visual Information Solutions, Boulder, Colorado). Optical density (OD) was defined as the sum of the RGB channels for the background light minus the sum of the RGB channels of each pixel. The values were normalized by dividing by $3 \times 255$. With this scale, OD varied between 0 (no color) and 1 (fully colored). Histologic evaluation of the investigated brain slices was conducted by a board-certified forensic pathologist who was blinded to the MR imaging findings.

\section{Image Registration}

A synthetic proton density-weighted image was generated from the $\mathrm{R}_{1}, \mathrm{R}_{2}$, and $\mathrm{PD}$ maps to show an image with good contrast between WM and GM. The histologic photographs were manually registered to the proton density-weighted image by using rotation, translation, and scaling. For this process, both quantification maps and photographs were resampled by interpolation to
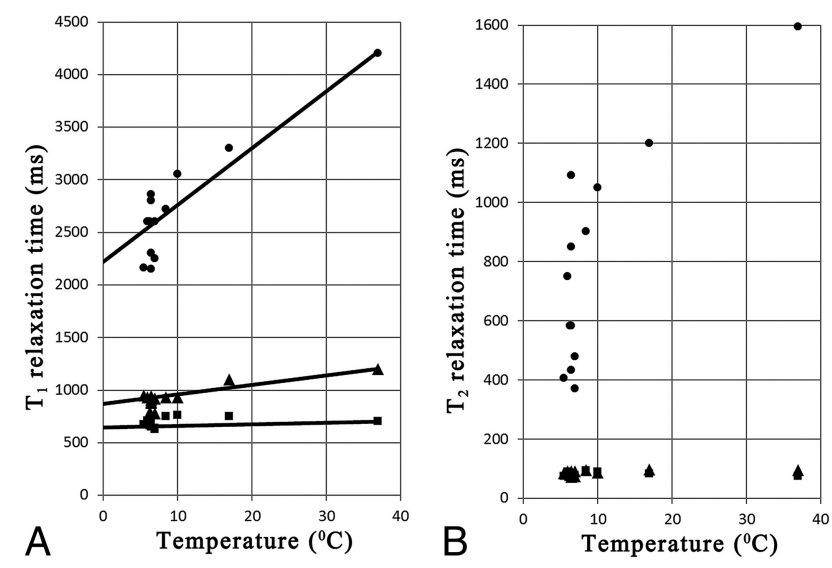

FIG 2. $A$, The observed mean $\mathrm{T} 1$ relaxation time in frontal white matter (squares), cortical gray matter (triangles), and ventricular CSF (dots) as a function of the core body temperature of all subjects. Added are the estimated slopes of $T 1$ change per temperature. $B$, The observed mean $\mathrm{T} 2$ relaxation time in frontal WM (squares), cortical GM (triangles), and ventricular CSF (dots) as a function of core body temperature of all subjects.

a resolution of $0.1 \mathrm{~mm} /$ pixel. The final registered maps and photographs were down-sampled again to the original MR image resolution of $0.7 \mathrm{~mm} /$ pixel to prevent an excessive number of data points for the correlation. In the down-sampling procedure, zeroes were excluded - that is, each pixel in the $0.7-\mathrm{mm} /$ pixel image was mapped to an area of $7 \times 7$ pixels of the $0.1-\mathrm{mm} /$ pixel image, where the value was calculated by the sum of the $7 \times 7$ pixels, divided by the number of pixels with a value larger than zero. By excluding zeroes, we avoided smoothing effects at the edges and cracks of the histologic specimen.

\section{Statistics}

Linear regression was performed on the LFB-OD as a function of $\mathrm{R}_{1}, \mathrm{R}_{2}, \mathrm{PD}$, and MR imaging myelin partial volume estimation for all registered pixels, excluding the value zero. The slope and intercept together with Spearman ranked correlation coefficient (Spearman $\rho$ ) were recorded for each subject and then averaged for the entire group. To visualize these $>100,000$ data points, we plotted them as 2D histograms of $40 \times 40$ bins, similar to plots in Engström et al. ${ }^{15}$ The intensity of the $2 \mathrm{D}$ histograms corresponds to the number of times a value occurs within each value interval. For each 2D histogram, 2 positions of highest density were determined to provide an estimate of the positions of GM and WM. These positions were averaged for the entire group. The line through the 2 positions was used as a second estimate of slope and intercept.

\section{RESULTS}

\section{Temperature Correction}

The mean T1 values for frontal WM, cortical GM, and ventricular CSF for the different temperatures are plotted in Fig $2 A$. Slopes were estimated at $1.5 \mathrm{~ms} /{ }^{\circ} \mathrm{C}$ for $\mathrm{WM}, 9.0 \mathrm{~ms} /{ }^{\circ} \mathrm{C}$ for $\mathrm{GM}$, and 54 $\mathrm{ms} /{ }^{\circ} \mathrm{C}$ for CSF. The intersection point for all 3 slopes was determined at $\mathrm{T} 1=600 \mathrm{~ms}$ and temperature $(\mathrm{T})=-30^{\circ} \mathrm{C}$. With this point as an origin, all observed $\mathrm{T} 1$ values at lower temperatures $\left(\mathrm{T}_{1, \mathrm{obs}}\right)$ in the entire acquisition volume were corrected for temperature (obtaining $\mathrm{T}_{1, \text { cor }}$ ) by using 


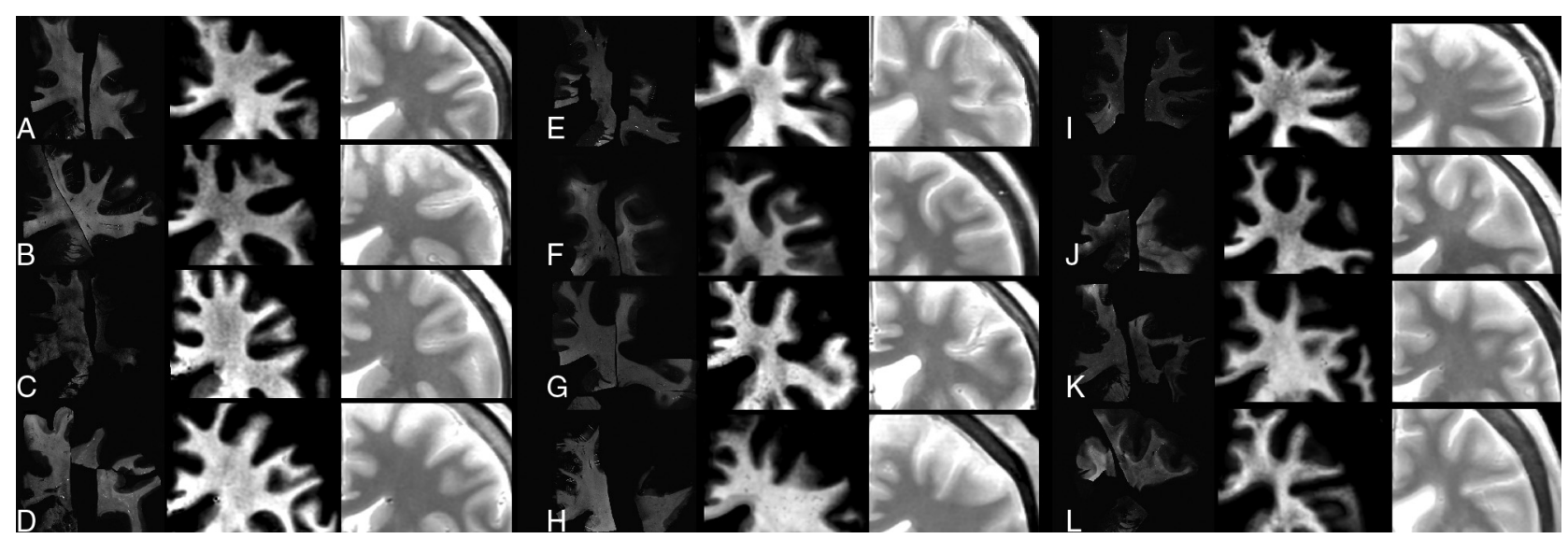

FIG 3. Zoomed-in images of all subjects, ordered according to increasing age $(A, 46 ; B, 47 ; C, 52 ; D, 55 ; E, 59 ; F, 61 ; G, 69 ; H, 70 ; I, 70 ; J, 71 ; K, 72 ;$ and $L, 74$ years of age). On the left, the registered LFB stains are shown as optical density, in the center is the MR imaging-detected myelin partial volume, and on the right is the synthetic PD-weighted images.

Table 1: The mean and SD for all subjects of the intercept and slope ${ }^{\mathrm{a}}$

\begin{tabular}{lccc}
\hline & Intercept & \multicolumn{1}{c}{ Slope } & Spearman $\boldsymbol{\rho}$ \\
\hline $\mathrm{R}_{1}$ & $1.19 \pm 0.10 \mathrm{~s}^{-1}$ & $0.065 \pm 0.009 \mathrm{~s}^{-1} / \%$ & $0.63 \pm 0.12$ \\
$\mathrm{R}_{2}$ & $12.31 \pm 1.17 \mathrm{~s}^{-1}$ & $0.044 \pm 0.131 \mathrm{~s}^{-1} / \%$ & $0.11 \pm 0.28$ \\
$\mathrm{PD}$ & $71.96 \pm 4.22 \%$ & $-2.45 \pm 0.53 \% / \%$ & $-0.73 \pm 0.09$ \\
Myelin & $1.50 \% \pm 2.84 \%$ & $4.37 \% \pm 1.73 \% / \%$ & $0.74 \% \pm 0.11$ \\
\hline
\end{tabular}

${ }^{a}$ Estimated by linear regression and the Spearman $\rho$ of the LFB optical density as a function of (uncorrected) $R_{1}$ and $R_{2}$ relaxation, proton density, and (temperaturecorrected) MRI-estimated myelin partial volume.

$$
T_{1, \mathrm{cor}}=600+\frac{T_{1, \mathrm{obs}}-600}{0.015 \times(T+30)} .
$$

For the lowest temperature at $\mathrm{T}=5.5^{\circ} \mathrm{C}$, the temperature correction corresponds to a $7.2 \%$ increase for WM T1 values, a $30.8 \%$ increase for GM T1 values, and a $67.6 \%$ increase for CSF T1 values.

The mean T2 values are plotted in Fig 2B. No clear trends were observed for changes of $\mathrm{T} 2$ as a function of temperature for WM or GM. The mean T2 for WM for all subjects was $80.5 \pm 8.0 \mathrm{~ms}$; the mean T2 for GM for all subjects was $87.2 \pm 8.3 \mathrm{~ms}$. The T2 values for CSF decrease with decreasing temperatures, but these exhibit a large variation. For this study, no T2 correction for temperature was made. Hence, the input values for the myelin model were $\mathrm{R}_{1, \text { cor }}=1 / \mathrm{T}_{1, \text { cor }}, \mathrm{R}_{2}=1 / \mathrm{T} 2$ and PD.

\section{Correlation of Estimated Myelin Partial Volume with LFB Optical Density}

In Fig 3, a zoomed-in image is shown of all the subjects, including the registered myelin estimation with histology staining (left column) and the myelin estimation with the qMRI method (center column). Visual inspection showed a good correspondence between the 2, though in some cases, the variability of the LFB method is evident. For example, in Fig 3B, 2 histologic specimens are positioned close together, showing an obviously different overall intensity. Also, peripheral areas (in eg, Fig $3 D,-K$, and $-L$ ) show an enhancing LFB-OD gradient.

In Table 1, the mean and SD of the intercept, slope, and Spearman $\rho$ of $R_{1}$ and $R_{2}$ relaxation, proton density, and MR imagingestimated myelin partial volume are given as a function of the LFB-OD. The Spearman $\rho$ shows a correlation for all parameters; weakest for $\mathrm{R}_{2}$ and stronger for $\mathrm{R}_{1}, \mathrm{PD}$, and myelin. The observed slope of myelin partial volume and LFB-OD was $4.37 \% \pm 1.73 \% / \%$; the intercept was $1.50 \% \pm 2.84 \%$.

All pixels $(>0)$ were plotted with $2 \mathrm{D}$ histograms, as shown in Fig 4 , where LFB-OD is taken as the $\mathrm{x}$-axis, and $\mathrm{R}_{1}, \mathrm{R}_{2}, \mathrm{PD}$, and qMRI-based myelin, as the y-axis. In Table 2, the mean and SD of the 2 positions of highest density in the $2 \mathrm{D}$ histograms are listed, attributed to WM and GM. The line through these 2 positions provides another way of estimating the slope and intercept, as listed in Table 2. The estimated slopes and intercepts in Table 2 are very similar to the values observed in Table 1 .

\section{Histologic Examinations}

In each of the 12 cases, histologic signs of slight cerebral edema (slight distension of perivascular and pericellular spaces, slight rarefaction of subpial spaces, and slight vacuolar appearance of gray matter neuropil) were present. Apart from that, no remarkable findings were seen.

\section{DISCUSSION}

In this work, a postmortem comparison was made between brain specimens dyed with myelin-sensitive Luxol fast blue and a qMRI method for estimating myelin. A challenging issue for postmortem MR imaging is to limit the influence of postmortem changes on the brain tissue, due to, for example, cause of death, the duration of the dying process, temperature, lack of blood flow, decay processes, and so forth. In our case, the cadavers were fresh and quickly refrigerated after death to limit changes due to decay processes. The brains were intact, and hence no effects of brain extraction or preservation played a role. However, all investigated brains showed histologic signs of slight cerebral edema, even though the brains appeared unremarkable at gross examination during the following postmortem examination. The reason for the appearance of slight cerebral edema may have been hypoxia during agony, despite all cases having causes of death that did not primarily affect the brain. Agony can last at least several minutes, which may be enough time to cause hypoxia in the brain due to deprived circulation and blood oxygenation. ${ }^{16-18}$

The presence of various degrees of edema most likely caused the near-zero slope in $R_{2}$ values (Table 1 ), which is much lower in 

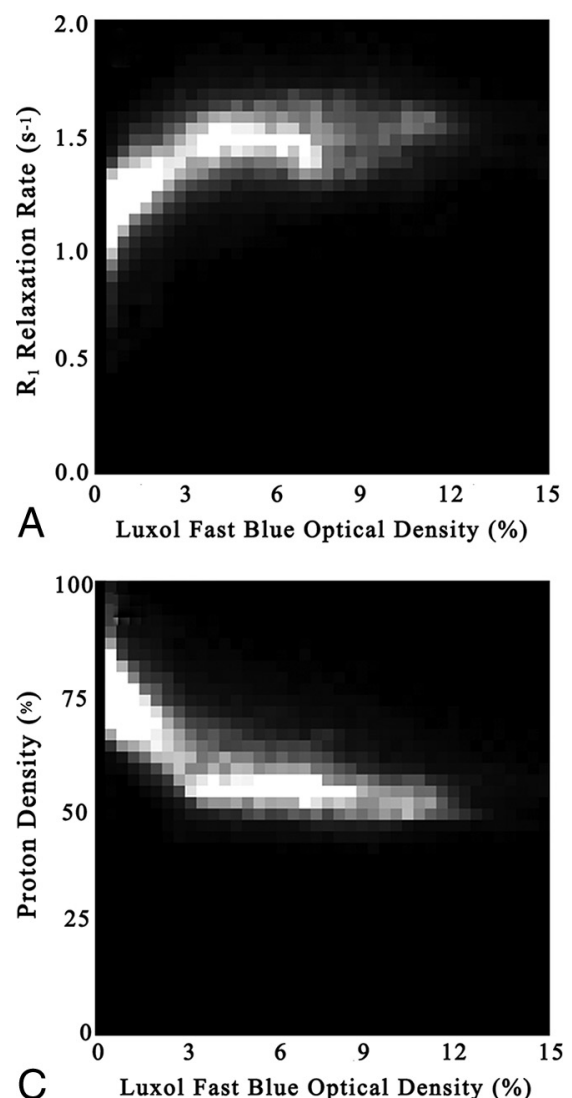

C Luxol Fast Blue Optical Density (\%)

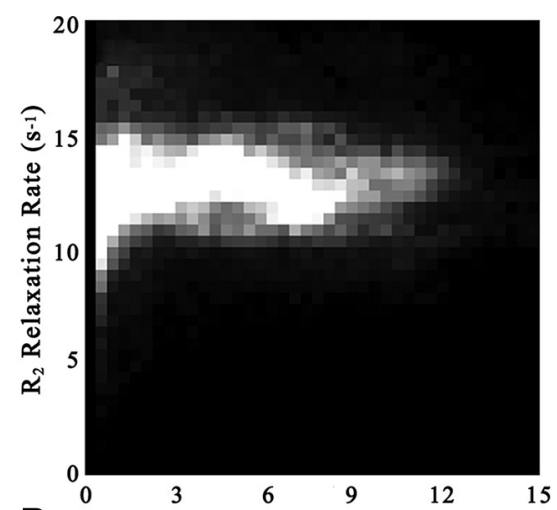

B Luxol Fast Blue Optical Density (\%)

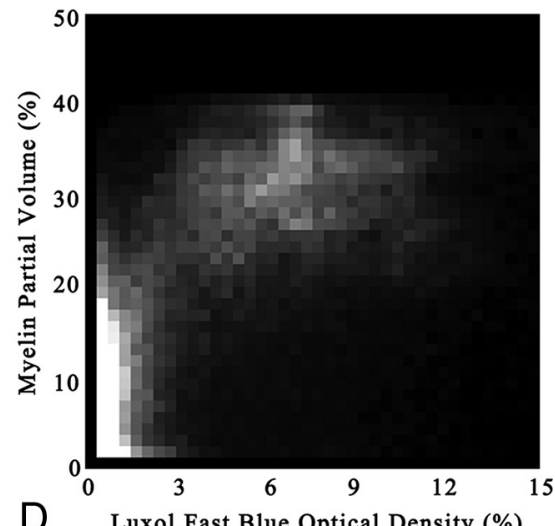

Luxol Fast Blue Optical Density (\%)

FIG 4. $2 D$ histograms of all subjects of the Luxol fast blue optical density values, plotted as a function of the observed $R_{1}$ relaxation rate $(A), R_{2}$ relaxation rate $(B)$, and proton density $(C)$. Also, the estimated myelin partial volume, based on temperature-corrected $R_{1}, R_{2}$, and PD is plotted $(D)$. The intensity of the $2 \mathrm{D}$ histograms corresponds to the number of times a value occurs within each value interval. Intervals were divided into $40 \times 40$ bins.

Table 2: The mean and SD for all subjects of the position of highest value density for white matter and gray matter observed in the 2D histograms ${ }^{a}$

\begin{tabular}{lcccc}
\hline & WM & GM & Intercept & Slope \\
\hline $\mathrm{R}_{1}$ & $1.47 \pm 0.08 \mathrm{~s}^{-1}$ & $1.27 \pm 0.09 \mathrm{~s}^{-1}$ & $1.24 \mathrm{~s}^{-1}$ & $0.030 \mathrm{~s}^{-1} / \%$ \\
$\mathrm{R}_{2}$ & $12.55 \pm 1.25 \mathrm{~s}^{-1}$ & $12.11 \pm 1.08 \mathrm{~s}^{-1}$ & $12.06 \mathrm{~s}^{-1}$ & $0.066 \mathrm{~s}^{-1} / \%$ \\
$\mathrm{PD}$ & $52.01 \% \pm 2.98 \%$ & $71.36 \% \pm 3.95 \%$ & $73.46 \%$ & $-2.85 \% / \%$ \\
Myelin & $30.98 \% \pm 3.77 \%$ & $4.84 \% \pm 2.30 \%$ & $2.01 \%$ & $3.84 \% / \%$ \\
LFB & $7.54 \% \pm 2.06 \%$ & $0.74 \% \pm 0.65 \%$ & - & - \\
\hline
\end{tabular}

${ }^{a}$ Added are the derived slopes and intercepts when using the positions of $R_{1}, R_{2}, P D$, and myelin as a function of the position of LFB.

comparison with the slope observed in the living. ${ }^{19,20}$ Also, the relatively large $S D$ of the $R_{2}$ slope indicates the sensitivity of $R_{2}$ values to changes in the brain. Peculiar is the observation of lower PD values than in living subjects (intercept of $71.96 \%$ ), but this may be a scaling issue in postprocessing. An important aspect of our data is the temperature of the cadaver. The mean core body temperature of the 12 subjects was $7.8^{\circ} \mathrm{C}$, which reduces the $\mathrm{T} 1$ and $\mathrm{T} 2$ relaxation times. The largest reduction was seen for CSF, whereas this occurred to a lesser extent for brain tissue. To get reasonable input data for the myelin model, we made a temperature correction only on the $\mathrm{T} 1$ relaxation times. The observed changes in $\mathrm{T} 1$ as a function of temperature were in line with those in previous reports, in which a linear behavior in $\mathrm{T} 1$ was shown for brain tissue. ${ }^{13,14,20}$ The observed slopes, however, were about half of the reported values from Birkl et $\mathrm{al}^{20}\left(1.5\right.$ versus $3 \mathrm{~ms} /{ }^{\circ} \mathrm{C}$ for WM and 9 versus $17 \mathrm{~ms} /{ }^{\circ} \mathrm{C}$ for $\left.\mathrm{GM}\right)$. With the temperature

correction, the input data to the myelin model were similar to those for living patients, in as much as possible for the deceased.

Concerning the LFB stains, the LFB-OD is not a quantitative measure, and the final OD values depend on the details of the staining procedure. Care was taken to keep all conditions as similar as possible, but color differences can occur due to small dye concentration differences, timing differences, or variation in sample thickness. The OD values of GM and WM in all subjects were within a reasonable interval for natural variation, but as shown in Fig 3, the staining method does not always show a uniform performance. The relatively large SD of the myelin slope $(1.73 \% / \%$; corresponding to $40 \%$ of the mean value of $4.37 \% / \%$ ) indicates the variability between subjects, and it is likely that issues with staining uniformity also affected the within-subject correlation and Spearman $\rho$.

Registration of LFB-stained brain slice photographs to MR images is a challenging ordeal. The orientation of the cuts is generally not identical to the MR images, and the fixation process of brain slices leads to shrinkage, cracks, and different kinds of distortions of the samples. $^{21,22}$ Moreover, there is a tremendous difference in the resolution of MR imaging and photographs of LFB-stained tissue specimens. Each pixel in an MR image corresponds (in our case) to $0.7 \times 0.7 \times 4.0 \mathrm{~mm}$, whereas each pixel in a photograph corresponds to $0.02 \times 0.02 \times 0.004 \mathrm{~mm}$. The difference in sharpness, in combination with imperfect registration, will cause substantial issues with partial volume at tissue interfaces, randomly spreading the data points in the correlation plots. The resolution mismatch will affect the regression analysis and probably lower the values of the Spearman $\rho$. The 2 approaches to retrieve the slopes (Tables 1 and 2), however, provided similar results. It can be speculated that the correlation values would improve by the application of ROIs to avoid tissue interfaces. This would, however, introduce a user dependency in the method, which we wanted to avoid.

A correlation was observed between LFB myelin staining and the qMRI-derived myelin estimation. The qMRI sequence is not sufficiently fast to resolve the short relaxation of myelin water directly, but the presence of myelin partial volume is inferred by its magnetization exchange effect on the (slower) cellular $\mathrm{R}_{1}$ and $\mathrm{R}_{2}$ relaxation rate components, as well as the decrease in observable proton density. The correlation of LFB-OD with the qMRIderived myelin detection shows a higher value $(\rho=0.74 \pm 0.11)$ than separate correlations with $\mathrm{R}_{1}, \mathrm{R}_{2}$, and $\mathrm{PD}(\rho=0.63 \pm 0.12$, $0.11 \pm 0.28$, and $0.73 \pm 0.09$, respectively, Table 1$)$. This finding supports the notion that the model can extract information from 
all 3 parameters to determine a myelin estimate. Most intriguing though are the substantially higher values for $\mathrm{R}_{1}$ and $\mathrm{PD}$ than for $R_{2}$, but these may be due to the mentioned postmortem edema effect. An indirect measurement such as this may have its limitations in comparison with a more direct multicomponent approach, but for clinical use, the robustness of the measurement may be more important. The qMRI method used is, for example, insensitive for $\mathrm{B}_{1}$ field inhomogeneity and radiofrequency pulse profile imperfections, ${ }^{9}$ and repeatability studies have shown a very low error in measured volume, for example $0.14 \%$ in brain size. $^{23}$ The clinical usefulness of the method is further supported by initial observations by Hagiwara et $\mathrm{al},{ }^{24}$ in which significant differences were found among normal-appearing white matter, MS plaque, and periplaque white matter in a group of patients with MS.

Our results show that myelin exhibits a nonsignificant but positive intercept with LFB-OD, suggesting that the myelin model may indicate values for GM that are higher in comparison with the LFB-OD. This finding is surprising because the expected limitations of both methods would rather lead to a negative intercept, in which qMRI-derived myelin in GM is measured at lower values than the LFB-OD. The MR imaging method is sensitive to the changes in the relaxation of intra- and extracellular water due to the proximity of fast relaxing, thin layers of myelin water, which are trapped between the myelin sheaths. Hence, it is not a direct measurement of myelin but rather a detection method of the thin-layered structure of the heavily myelinated nerve fibers. If axons or dendrites in GM are insulated with a single sheath, no water can be trapped in thin layers and the MR imaging method will cease to measure the presence of myelin. An issue for the LFB staining, on the other hand, is that the dye targets lipoproteins; hence, it may also stain substances other than myelin, such as neuropil, the cell membranes, and nuclei of nerve cells. This staining may create a small, positive offset in optical density, for example, as observed by Laule et al. ${ }^{25,26}$ Possibly, the explanation lies in the noise of the MR imaging measurements: Detection of a higher myelin value due to noise is allowed, whereas there can be no values lower than zero. Low myelin values may therefore exhibit a small rectification offset.

A limitation of the study is the low number of cases included. Also, no specific brain pathology such as multiple sclerosis or dementia was investigated. Full clinical medical records of the subjects were not made available to the study due to the forensic nature of cases. However, the available preliminary information did not reveal the existence of relevant neurologic illnesses. Moreover, gross brain examinations at postmortem examination and histologic examinations of investigated brain slices did not present relevant pathology. For future work, it would be very interesting to repeat the study for specific patient groups with relevant demyelination pathologies, microangiopathy, or lacunar infarcts, provided it is practically possible to retrieve full medical history in the short time between death and investigation.

In the histologic investigation, evidence of edema was found. Edema can alter relaxation times ${ }^{27,28}$ and hence will have an effect on the myelin detection. The quantification of edema is included in the qMRI model used, but there is no straightforward method of histologically mapping edema, precluding a possible correlation analysis for this study.

\section{CONCLUSIONS}

A correlation was shown between a myelin-detection method based on qMRI and myelin staining of brain slice by using Luxol fast blue in a postmortem setting, supporting the validity of myelin measurement with the qMRI method.

Disclosures: Marcel Warntjes—UNRELATED: Employment: SyntheticMR AB, Comments: part-time employment; Stock/Stock Options: SyntheticMR AB.

\section{REFERENCES}

1. Miller DH, Barkhof F, Frank JA, et al. Measurement of atrophy in multiple sclerosis: pathological basis, methodological aspects and clinical relevance. Brain 2002;125:1676-95 CrossRef Medline

2. Bakshi R, Thompson AJ, Rocca MA, et al. MRI in multiple sclerosis: current status and future prospects. Lancet Neurol 2008;7:615-25 CrossRef Medline

3. Ihara M, Polvikoski TM, Hall R, et al. Quantification of myelin loss in frontal lobe white matter in vascular dementia, Alzheimer's disease, and dementia with Lewy bodies. Acta Neuropathol 2010;119: 579-89 CrossRef Medline

4. Back SA, Riddle A, McClure MM. Maturation-dependent vulnerability of perinatal white matter in premature birth. Stroke 2007;38: 724-30 CrossRef Medline

5. Croteau-Chonka EC, Dean DC 3rd, Remer J, et al. Examining the relationships between cortical maturation and white matter myelination throughout early childhood. Neuroimage 2016;125:413-21 CrossRef Medline

6. MacKay A, Laule C, Vavasour I., et al. Insights into brain microstructure from the T2 distribution. Magn Reson Imag 2006;24: 515-25 CrossRef Medline

7. Oh J, Han ET, Lee MC, et al. Multislice brain myelin water fractions at 3T in multiple sclerosis. J Neuroimaging 2007;17:156-63 CrossRef Medline

8. Deoni SC, Rutt BK, Arun T, et al. Gleaning multicomponent T1 and T2 information from steady-state imaging data. Magn Reson Med 2008;60:1372-87 CrossRef Medline

9. Levesque IR, Pike GB. Characterizing healthy and diseased white matter using quantitative magnetization transfer and multicomponent T(2) relaxometry: A unified view via a four-pool model. Magn Reson Med 2009;62:1487-96 CrossRef Medline

10. Alonso-Ortiz E, Levesque IR, Pike GB. MRI-based myelin water imaging: a technical review. Magn Reson Med 2015;73:70-81 CrossRef Medline

11. Warntjes M, Engström M, Tisell A, et al. Modelling the presence of myelin and edema in the brain based on multi-parametric quantitative MRI. Front Neurol 2016;7:16 CrossRef Medline

12. Warntjes JBM, Dahlqvist $O$, West J, et al. Rapid magnetic resonance quantification on the brain: optimization for clinical usage. Magn Reson Med 2008;60:320-29 CrossRef Medline

13. Tashiro K, Shiotani S, Kobayashi T, et al. Cerebral relaxation times from postmortem MR imaging of adults. Magn Reson Med Sci 2015; 14:51-56 CrossRef Medline

14. Zech WD, Hottinger AL, Schwendener N, et al. Post-mortem 1.5T MR quantification of regular anatomical brain structures. Int $\mathrm{J} \mathrm{Le}$ gal Med 2016;130:1071-80 CrossRef Medline

15. Engström M, Warntjes JB, Tisell A, et al. Multi-parametric representation of voxel-based quantitative magnetic resonance imaging. PLoS One 2014;9:e111688 CrossRef Medline

16. Hausmann R, Vogel C, Seidl S, et al. Value of morphological parameters for grading of brain swelling. Int J Legal Med 2006;120:219-25 CrossRef Medline

17. Dettmeyer RB. Forensic Neuropathology: Forensic Histopathology. Berlin: Springer; 2011:413-438 
18. Oehmichen M, Auer RN, König HG. Cell and tissue reactions. In: Oehmichen M, Auer RN, König HG. Forensic Neuropathology and Associated Neurology. Berlin: Springer; 2006:42-76

19. Warntjes JB, Engström M, Tisell A, et al. Brain characterization using normalized quantitative magnetic resonance imaging. PLoS One 2013;8:e70864 CrossRef Medline

20. Birkl C, Langkammer C, Haybaeck J, et al. Temperature dependency of T1 relaxation time in unfixed and fixed human brain tissue. NMR Biomed 2016;29:458-65

21. Moore GR, Leung E, MacKay AL, et al. A pathology-MRI study of the short-T2 component in formalin-fixed multiple sclerosis brain. Neurology 2000;55:1506-10 CrossRef Medline

22. Bronge L, Bogdanovic N, Wahlund LO. Postmortem MRI and histopathology of white matter changes in Alzheimer brains: a quantitative, comparative study. Dement Geriatr Cogn Disord 2002;13:205-12 CrossRef Medline

23. Granberg T, Uppman M, Hashim F, et al. Clinical feasibility of synthetic MRI in multiple sclerosis: a diagnostic and volumetric validation study. AJNR Am J Neuroradiol 2016;37:1023-29 CrossRef Medline
24. Hagiwara A, Hori M, Yokoyama K, et al. Utility of a multiparametric quantitative MRI model that assesses myelin and edema for evaluating plaques, periplaque white matter, and normal-appearing white matter in patients with multiple sclerosis: a feasibility study. AJNR Am J Neuroradiol 2017;38:237-42 CrossRef Medline

25. Laule C, Leung E, Lis DK, et al. Myelin water imaging in multiple sclerosis: quantitative correlations with histopathology. Mult Scler 2006;12:747-53 CrossRef Medline

26. Laule C, Vavasour IM, Leung E, et al. Pathological basis of diffusely abnormal white matter: insights from magnetic resonance imaging and histology. Mult Scler 201 1;17:144-50 CrossRef Medline

27. Neema M, Stankiewicz J, Arora A, et al. T1- and T2-based MRI measures of diffuse gray matter and white matter damage in patients with multiple sclerosis. J Neuroimaging 2007;17:16S-21S CrossRef Medline

28. Larsson HB, Frederiksen J, Petersen J, et al. Assessment of demyelination, edema and gliosis by in-vivo determination of $\mathrm{T} 1$ and $\mathrm{T} 2 \mathrm{in}$ the brain of patients with acute attack of multiple sclerosis. Magn Reson Med 1989;11:337-48 Medline 